\title{
Thin-Film Polyimide Membrane Actuators Fabricated by Etching a Substrate by DRIE (Bosch Process)*
}

\author{
Satomitsu IMAI**, Yusuke SHIMIZU** and Shingo MATSUNO** \\ **Department of Precision Machinery Engineering, Nihon University, \\ 7-24-1 Narashinodai, Funabashi-shi, Chiba 274-8501, Japan \\ E-mail: imai@eme.cst.nihon-u.ac.jp
}

\begin{abstract}
This paper deals with thin-film polyimide membrane actuators. The membranes were fabricated by deep-RIE using the Bosch process for etching a silicon substrate up to the polyamide layer, since we found that the polyimide layer was scarcely etched in this process. Membranes that were $5 \mathrm{~mm}$ in diameter and 2-10 $\mu \mathrm{m}$ thick were fabricated. The characteristics of membrane deflection were experimentally and analytically investigated by applying static pressure. The membranes could produce displacement on the order of a hundred micrometers in an experiment. We chose thermal expansion to actuate the membranes as actuators. Applying electricity to an aluminum layer deposited on the membrane deflected the membrane due to the thermal expansion of the aluminum layer. The experiments demonstrated that membranes 3-10 $\mu \mathrm{m}$ thick produced displacements at the membrane centers in a range from $10-20 \mu \mathrm{m}$ by applying $1 \mathrm{~V}$ and the actuators worked periodically by turning the supply of electricity on and off.
\end{abstract}

Key words: Polyimide, Membrane, Thin Film, MEMS, Deep RIE, Bosch Process, Actuator, Thermal Expansion

\section{Introduction}

Polyimide (PI) membranes have been widely used in Micro-electro-mechanical systems (MEMS) based on its low elastic modulus, good mechanical properties, high electric isolation, and high heat resistance ${ }^{(1)-(6)}$. A typical method to fabricate a polyimide membrane has been removing the substrate on which a polyimide layer is coated by wet etching ${ }^{(1)-(4)}$. In general, an etch-stop layer has also been used in this method. However, this method has problems that fabricating an arbitral shape of the membrane is impossible and fabrication process is not simple. In addition, the etch-stop layer increases membrane stiffness if it is not removed. The purpose of this study is to develop a simple fabrication method of thin-film polyimide membranes by dry etching without using an etch-stop layer and to apply the membranes fabricated with this method to MEMS actuators. Typical examples of fabricating a polyimide membrane in conventional studies are as follows. Shearwood et al. fabricated polyimide membranes $(\phi 1-8 \mathrm{~mm}, \mathrm{t} 7 \mu \mathrm{m})$ by wet etching to remove a GaAs substrate ${ }^{(1)}$. Nara et al. used wet etching for an Si substrate ${ }^{(2)}$. An etch-stop layer $\left(\mathrm{Si}_{3} \mathrm{~N}_{4}\right)$ has also been used (membranes: $4 \times 1 \mathrm{~mm}, \mathrm{t} 1.3 \mu \mathrm{m}$ ). Lin et al. used wet etching for an $\mathrm{Si}$ substrate and an etch-stop layer $\left(\mathrm{SiO}_{2}\right)$ has also been used (membranes: $\phi$ $20 \mathrm{~mm}, \mathrm{t} 2.15 \mu \mathrm{m}){ }^{(3)}$. Gowrishetty et al. used DRIE for etching an Si substrate ${ }^{(5)}$. Their method was similar to ours. However, they used an etch-stop layer $\left(\mathrm{SiO}_{2}\right)$ and it was not removed, i.e., their membranes consisted of a polyimide layer and an etch-stop layer (membranes: $\phi 0.3 \mathrm{~mm}, \mathrm{t} 2.5 \mu \mathrm{m}$ ).

Received 26 Sep., 2011 (No. 11-0582) [DOI: 10.1299/jamdsm.6.287]

Copyright $\odot 2012$ by JSME 
In our method, we specifically spin-coated a polyimide layer on a silicon ( $\mathrm{Si}$ ) substrate and then removed a membrane section of the substrate by deep reactive ion etching (DRIE) using the Bosch process. We confirmed from an experiment that polyimide layers were scarcely etched with this method. Therefore, thin-film polyimide membranes with arbitrary shapes could easily be fabricated without any etch-stop layer for the etching process. We could fabricate polyimide membranes that were $5 \mathrm{~mm}$ in diameter and 2-10 $\mu \mathrm{m}$ thick with the method we propose. These thicknesses are comparable with those in conventional studies ${ }^{(1)-(5)}$. However, we also found a problem with this method in that very thin polyimide layers were damaged in the Bosch process. The experiment we carried out demonstrated that the minimum thickness of the polyimide membrane was $2 \mu \mathrm{m}$.

The characteristics of deflection of the membrane we fabricated were investigated. First, the membrane deflections were measured by applying static pressure ${ }^{(2),(5)}$. The experiments revealed that the maximum displacement of the membrane center was in a range of several hundreds of micrometers for membranes that were 2-10 $\mu \mathrm{m}$ thick. The polyimide membranes showed the large expandability. However, theoretical analysis conducted to assess the displacements in the experiment revealed that residual stress of the polyimide membrane decreased the generated displacement. This suggests that it is necessary to optimize process condition of the polyimide layer to decrease the residual stress. Next, thermal expansion by applying electricity was chosen as a driving method for the polyimide membranes to act as actuator ${ }^{(7),(8)}$. The reason is that polyimide has high electrical isolation and high heat resistance. Thin-film aluminum (Al) layers to apply electricity were deposited on the membranes. The characteristics of the displacement of the actuators were investigated by applying electrical voltage of $1 \mathrm{~V}$. The experiments indicated that the maximum displacements that the actuator could produce were $10-20 \mu \mathrm{m}$ for $3-10 \mu \mathrm{m}$ thick membranes and the actuators worked periodically for about a 50-s period by turning the input electricity on and off. We consider that this actuator can be available for MEMS such as micro-switches, micro-valves, and micro-positioning systems ${ }^{(2)-(5)}$.

\section{Structure and fabrication process for polyimide membranes}

\subsection{Structure and dimensions of membranes}

Figure 1 (a) shows the structure and dimensions of the membranes. There is a thin-film polyimide layer on the silicon substrate and the substrate is $0.4-\mathrm{mm}$ thick. The membrane is $5 \mathrm{~mm}$ in diameter and 2-10 $\mu \mathrm{m}$ thick. The thin aluminum layer deposited on the membrane is for applying electricity. Table 1 summarizes the properties of the materials we used. We used two kinds of polyamides as prepolymers of polyimide; PIX-3400 had normal viscosity and PIX-1400 had low viscosity. The properties in Table 1 are cited from supplier's data ${ }^{(9)}$. Figures 1 (b) is a photograph of the membrane we fabricated.

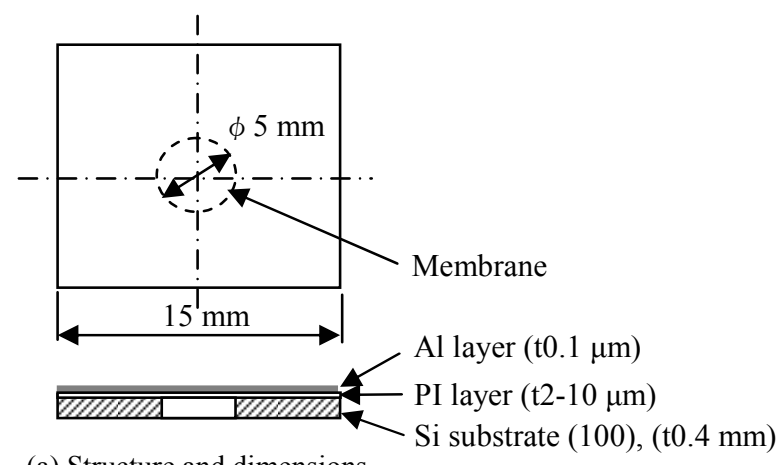

(a) Structure and dimensions

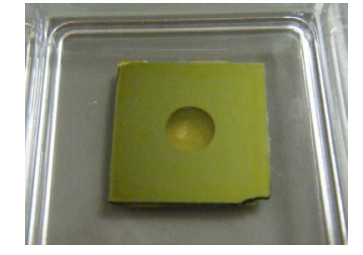

(b) Sample without Al layer

Fig. 1 Structure and dimensions of polyimide (PI) membranes. Figure (b) shows a photograph of the sample without the Al layer. This figure is a top view from the PI layer side. 
Table 1 Material properties

\begin{tabular}{|c|c|c|c|c|}
\hline & \multicolumn{2}{|c|}{ Polyamide } & \multirow{2}{*}{ Aluminum } & \multirow{2}{*}{$\begin{array}{c}\text { Silicon } \\
(100)\end{array}$} \\
\hline & PIX-3400* & PIX-1400* & & \\
\hline Density $\times 10^{3}\left[\mathrm{~kg} / \mathrm{m}^{3}\right]$ & 1.09 & 1.07 & 2.7 & 2.33 \\
\hline Viscosity $[\mathrm{Pa} \cdot \mathrm{s}]$ & 13.0 & 1.1 & - & - \\
\hline Modulus of elasticity [GPa] & \multicolumn{2}{|c|}{3.0} & 70 & 130 \\
\hline Poisson's ratio & \multicolumn{2}{|c|}{0.34} & 0.34 & - \\
\hline Tensile strength [MPa] & \multicolumn{2}{|c|}{150} & $(100)$ & - \\
\hline $\mathrm{CTE}^{* *} \times 10^{-6}\left[1 /{ }^{\circ} \mathrm{C}\right]$ & \multicolumn{2}{|c|}{50} & 23.8 & 2.33 \\
\hline
\end{tabular}

* Hitachi Chemical and DuPont ${ }^{(9)}$,** Coefficient of thermal expansion

\subsection{Fabrication processes}

The fabrication processes are schematically outlined in Fig. 2. First, an aluminum (Al) layer that was about $0.1-\mu \mathrm{m}$ thick was deposited on the silicon ( $\mathrm{Si}$ ) substrate. Then, the $\mathrm{Al}$ layer for the diaphragm section was patterned by using photolithography (Fig. 2(a)). This pattern was used as a mask for the deep-RIE (DRIE) that was conducted later. When the substrate thickness was less than about $0.2 \mathrm{~mm}$, a resin mask could also be used.

Polyamide as a prepolymer of polyimide was used for spin coating since polyimide is not a fluid. The polyamide was spin-coated on the substrate surface opposite to the Al mask. The film thickness of the polyamide was determined in relation to the spinning speed and viscosity. The PIX-1400 in Table 1 was used for polyimide layer thicknesses equal to or less than $2 \mu \mathrm{m}$. Then, the polyamide layer was baked to convert it into polyimide (Fig. 2(b)). Figure 3 shows the chemical bonds for the polyamide and polyimide. Table 2 lists the baking conditions. This process consisted of two steps of pre-baking and main-baking. The temperature for the main baking was about $350{ }^{\circ} \mathrm{C}$. These baking conditions were determined to prevent the polyimide layer from being damaged.

Then, the Si substrate was etched by using deep-RIE to obtain a membrane (Fig. 2(c)). The deep-RIE used the Bosch process, which can etch silicon substrates deeply and vertically by alternately repeating the etching and passivation processes. We tested and confirmed that this process scarcely etched the polyimide layer, i.e., this process did not need an etch-stop layer. Table 3 summarized the process conditions for the Bosch process (equipment: Sumitomo Precision Products, MUC-21). Arbitrary shapes of membranes could easily be formed with this method. The minimum membrane thickness that could be achieved with this approach was found to be $2 \mu \mathrm{m}$. The membranes were damaged in the Bosch process for thicknesses below $2 \mu \mathrm{m}$. We consider that it is necessary to optimize the etching conditions.

Finally, an Al layer to apply an electric current was deposited onto the polyimide membrane surface ${ }^{(7),(8)}$ (Fig. 2(d)). Vacuum vapor deposition was used to form this layer ${ }^{(3)}$, (6). The Al layer was about $0.1 \mu \mathrm{m}$ thick. This layer did not peel off even when pressure loads were applied in the experiments that were conducted later.

Table 4 summarizes the relationship between the fabricated membrane thicknesses and process conditions. The membrane thickness was obtained by the difference between the total thickness (the substrate and the polyimide layer) and the substrate thickness. The surface roughness of the polyimide membranes was also examined. The dry-etched sides of the membranes had larger surface roughness than those that had not been etched. This indicated that the sides exposed to the etching environment had been etched slightly. 


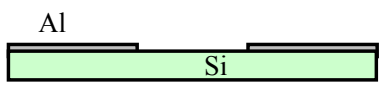

(a) Depositing aluminum layer and patterning it by photolithography

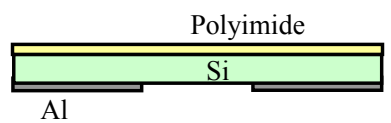

(b) Spin-coating polyamide and baking it to convert it into polyimide

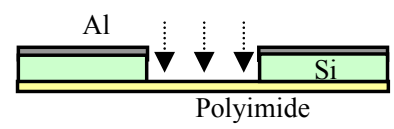

(c) Removing membrane section of Si substrate by deep-RIE using Bosch process

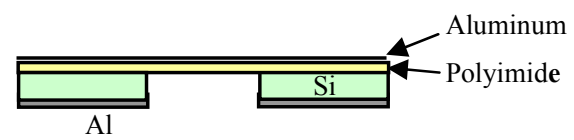

(d) Depositing aluminum layer to apply electricity to polyimide membrane

Fig. 2 Fabrication processes for polyimide membranes. Characteristic part in processes is membrane fabrication by removing Si substrate up to polyimide layer by using deep-RIE using Bosch process (c).

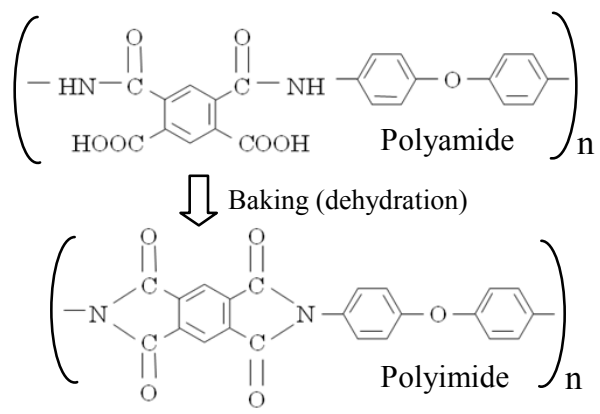

Table 2 Baking conditions for polyamide

\begin{tabular}{c|c|c}
\hline \multicolumn{2}{c|}{ Pre-bake } & $120^{\circ} \mathrm{C} / 5 \min$ (Oven) \\
\hline $\begin{array}{l}\text { Main } \\
\text { bake }\end{array}$ & 1st step & $200^{\circ} \mathrm{C} / 5 \min$ (Hot plate) \\
\hline
\end{tabular}

Fig. 3 Chemical bonds for polyamide and polyimide

Table 3 Process conditions for deep-RIE* (Bosch process)

\begin{tabular}{|c|c|c|}
\hline Gas & Passivation step & Etching step \\
\hline $\begin{array}{ll}\mathrm{SF}_{6} & {[\mathrm{sccm}]}\end{array}$ & - & 550 \\
\hline $\mathrm{O}_{2} \quad[\mathrm{sccm}]$ & - & 55 \\
\hline $\mathrm{C}_{4} \mathrm{~F}_{8} \quad[\mathrm{sccm}]$ & 350 & - \\
\hline Step time $\quad[\mathrm{s}]$ & 3 & 10 \\
\hline Gas pressure $[\mathrm{Pa}]$ & $4.4-5.4$ & $5.6-6.3$ \\
\hline
\end{tabular}

Table 4 Polyimide layer thickness, process conditions, and surface roughness

\begin{tabular}{|c|c|c|c|c|}
\hline \multirow{2}{*}{$\begin{array}{l}\text { Polyimide layer } \\
\text { thickness }[\mu \mathrm{m}]\end{array}$} & \multirow{2}{*}{$\begin{array}{c}\text { Polyamide } \\
\text { type }\end{array}$} & \multirow{2}{*}{$\begin{array}{c}\text { Spinning } \\
\text { speed }^{* *}[\mathrm{rpm}]\end{array}$} & \multicolumn{2}{|c|}{ Surface roughness of PI layer*** $(\mathrm{Ra})[\mu \mathrm{m}]$} \\
\hline & & & Etched side & Not etched side \\
\hline 10 & PIX-3400* & 2500 & 0.16 & 0.009 \\
\hline 6 & $\uparrow$ & 4000 & - & - \\
\hline 4 & $\uparrow$ & 5400 & 0.50 & 0.016 \\
\hline 2 & PIX-1400* & 2800 & - & - \\
\hline 1 & $\uparrow$ & 6000 & broken & - \\
\hline
\end{tabular}

* cf. Table $1, * *$ spinning time: 30 [s]

*** average values, measured with interferometry surface roughness meter (Taylor Hobson, Talysurf CCI Lite)

\section{Results from measurements}

\subsection{Characteristics of membrane displacement by applying pressure}

Figure 4 outlines the measurement setup to characterize displacement due to membrane deflection actuated by static air pressure ${ }^{(2),(5)}$. The pressure was applied with a manually operated cylinder. The displacement of the membrane center and the applied pressure were 
measured. The resolution of the laser displacement sensor was $0.05 \mu \mathrm{m}$ (Keyence, LK-G30) and that of the pressure manometer was $0.1 \mathrm{kPa}$ (Copal Electronics, PG-100-102GP).

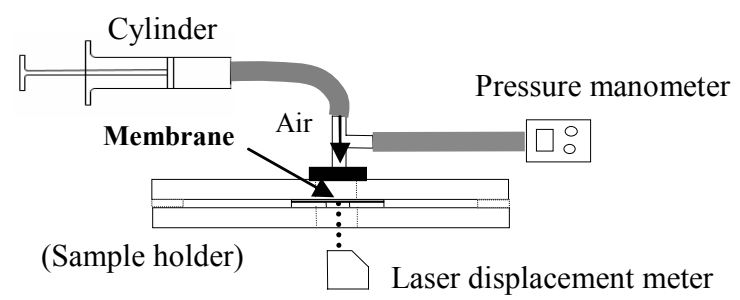

Fig. 4 Setup for measuring displacement of membrane center and applied air pressure. Membrane is deflected by applying air pressure.

Figures 5 (a) and (b) plot the relationship between the displacement of the membrane center and the applied pressure for 10- and $4-\mu \mathrm{m}$ thick membranes. The displacements of the membranes with and without the aluminum (Al) layer (Fig. 2 (a)) have been plotted in the figures. Figure 5 indicates that the displacement-pressure relationship has negligibly small hysteresis and that the deposited Al layer on the membrane did not affect the characteristics of displacement. This is probably because the layer was very thin $(\mathrm{t} 0.1 \mu \mathrm{m})$.

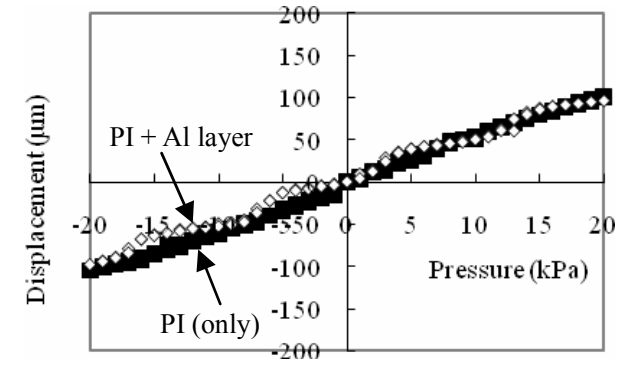

(a) Polyimide membrane thickness: $10 \mu \mathrm{m}$

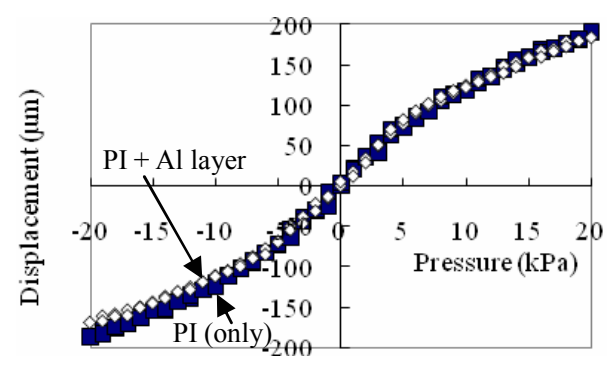

(b) Polyimide membrane thickness: $4 \mu \mathrm{m}$

Fig. 5 Relationship between applied pressure and displacement of membrane center. Polyimide (PI) membrane thicknesses are (a) $10 \mu \mathrm{m}$ and (b) $4 \mu \mathrm{m}$. Pressure varies on order of $0 \rightarrow 20 \rightarrow-20 \rightarrow 0 \mathrm{kPa}$ to examine hysterisis of membrane displacement.

\subsection{Theoretical analysis of membrane displacement}

Theoretical analysis of the displacements of the membrane center without the Al layer was conducted to assess the displacements in Fig. 5. Equation (1) was used to derive the displacement of a thin round membrane as a function of applied uniform pressure ${ }^{(10)}$. This equation is used when displacement is larger than the membrane thickness.

$$
d=0.662 r \sqrt[3]{\frac{p r}{E t}}
$$

where $d$ is the displacement of the membrane center, $r$ is the radius of the membrane, and $p$ is the applied pressure. Here, $E$ is Young's modulus for the membrane and $t$ is the membrane thickness.

Figures 6 (a) and (b) plot the analytical displacements for 10- and 4- $\mu \mathrm{m}$ thick membranes with respect to the applied pressure. The displacements were obtained by substituting the membrane dimensions (Fig. 1), the material properties (Table 1), and the measured pressure (Fig. 5) into Eq. (1). The curves in the figures indicate the analytical displacements and the closed squares plot the experimental results. The figures suggest that there are constant gaps between both results over the pressure range. We considered the reason for this to be residual stress (tensile) caused by baking (Fig. 2 (b)). 


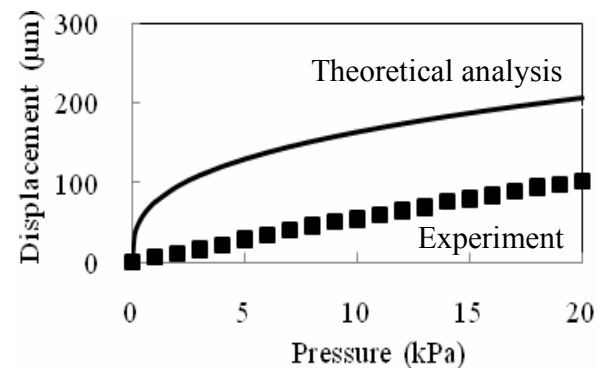

(a) Polyimide membrane thickness: $10 \mu \mathrm{m}$

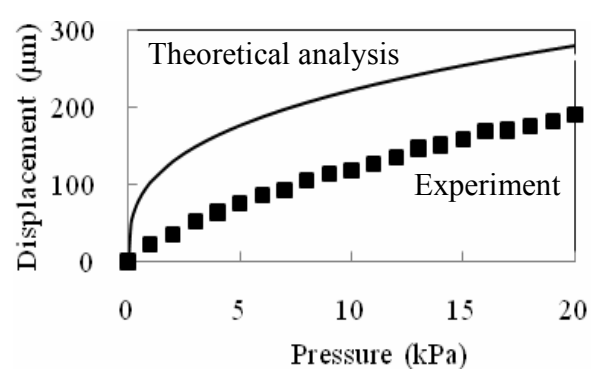

(b) Polyimide membrane thickness: $4 \mu \mathrm{m}$

Fig. 6 Comparison of experimentally measured and theoretically analyzed displacements of membrane center. Closed squares mean measured displacements and curves mean theoretically analyzed displacements calculated with Eq. (1).

Residual stress was investigated and Eq. (2) provides the theoretical pressure-deflection relationship when considering residual stress in the membrane ${ }^{(11)}$.

$$
p=4 \frac{t}{r} \frac{d}{r}\left(\sigma_{0}+\frac{2 E}{3(1-v)} \frac{d^{2}}{r^{2}}\right)
$$

where $\sigma_{0}$ is residual stress, $v$ is Poisson's ratio, and the other variables are the same as those in Eq. (1). The residual stress $\left(\sigma_{0}\right)$ can be obtained by substituting membrane dimensions, material properties, and the measured displacement and pressure into Eq. (2). The residual stresses calculated from the measured displacements are almost constant. The average values for the residual stresses we obtained are $26.9 \mathrm{MPa}$ for the $10-\mu \mathrm{m}$ thick membrane and 24.9 MPa for the 4- $\mu \mathrm{m}$ thick membranes. By taking into consideration these average values, the displacements recalculated by Eq. (2) come to fit well to the measured displacements over the whole measurement range. Figure 7 confirms this. The curves in the figure indicate the theoretical displacements and the closed squares and triangles plot the measured displacements. This figure also suggests that it is possible to obtain larger deflections of membranes by decreasing the residual stress.

\subsection{Maximum displacements of polyimide membranes}

The maximum displacements that the polyimide membranes could produce were examined. Figure 8 plots the pressure-displacements relationship when the applied pressure was increased up to the point where it burst the membrane. The membranes we tested were 3and 6- $\mu \mathrm{m}$ thick (without the Al layer in Fig. 1 (a)). The 10- $\mu \mathrm{m}$ thick membranes did not break in the pressure range we applied. Figure 8 demonstrates that the maximum displacements of the membrane center were on the order of several hundred micrometers.

The maximum stress for the membranes we tested was theoretically examined. Maximum stress occurs at the center of the membranes theoretically. Equation (3) was used to derive the stress at the center of membranes when uniform pressure was applied to their surfaces (10).

$$
\sigma=0.423 \sqrt[3]{E p^{2} \frac{r^{2}}{t^{2}}}
$$

where $\sigma$ is the maximum stress, and the other variables are the same as those in Eq. (1). The maximum stresses in the polyimide membranes due to the maximum pressures in Fig. 8 are calculated to be $164 \mathrm{MPa}$ for the $3-\mu \mathrm{m}$ thick membrane and $107 \mathrm{MPa}$ for the $6-\mu \mathrm{m}$ thick membrane by substituting the maximum pressures into Eq. (3). Residual stresses also exist in their membranes. The residual stress can be estimated with Eq. (2) as described in section $3 \cdot 2$, i.e., $33 \mathrm{MPa}$ for the $3-\mu \mathrm{m}$ thick membrane and $35 \mathrm{MPa}$ for the $6-\mu \mathrm{m}$ thick membrane. 
Therefore, the maximum stresses for the membranes in Fig. 8 are estimated to be $197 \mathrm{MPa}$ for the 3- $\mu \mathrm{m}$ thick membrane and $142 \mathrm{MPa}$ for the $6-\mu \mathrm{m}$ thick membrane. These values almost correspond to the data that the supplier suggests $(150 \mathrm{MPa} \text {, cf. Table } 1)^{(9)}$.

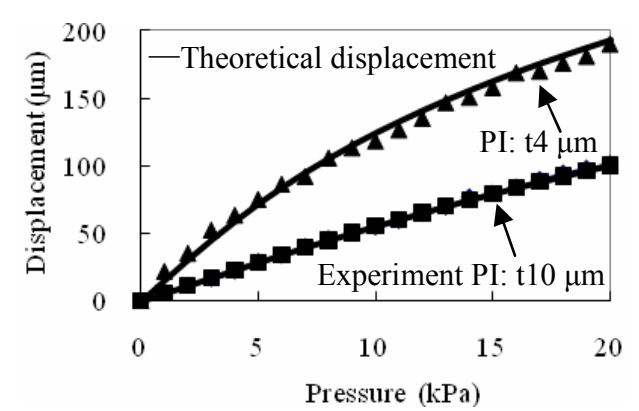

Fig. 7 Theoretical and measured displacements of membrane center. Theoretical displacements take into consideration residual stress.

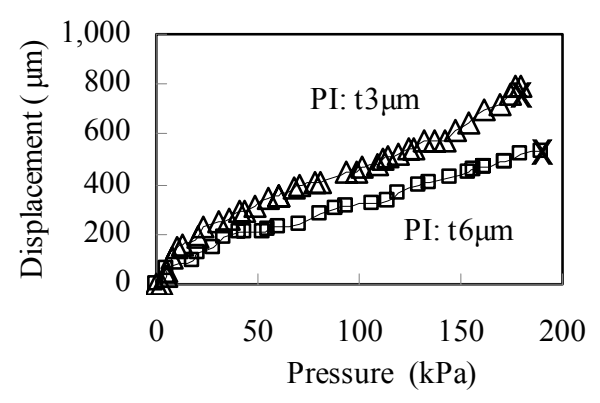

Fig. 8 Maximum displacements of membrane center when pressure applied to membranes are increased.

\subsection{Characteristics of membrane deflection driven by thermal expansion}

We chose thermal expansion to actuate the polyimide membrane as a driving method ${ }^{(7)}$, ${ }^{(8)}$. To do that, an aluminum ( $\mathrm{Al}$ ) layer (about $0.1-\mu \mathrm{m}$ thick) was deposited on the polyimide membrane ${ }^{(3),(6)}$. When an electric current was applied, the resulting thermal expansions of the Al layer and the polyimide layer generated membrane deflection. The coefficients of thermal expansion are listed in Table 1. Figure 9 outlines the setup we used to measure displacement of the membrane center. The temperature of the Al layer of the membrane center was also measured with a thermo-couple (AS ONE, TXN-400E, resolution: $\pm 2{ }^{\circ} \mathrm{C}$ ). The input voltage was $1 \mathrm{~V}$ and the resulting electric current was about $0.26 \mathrm{~A}$.

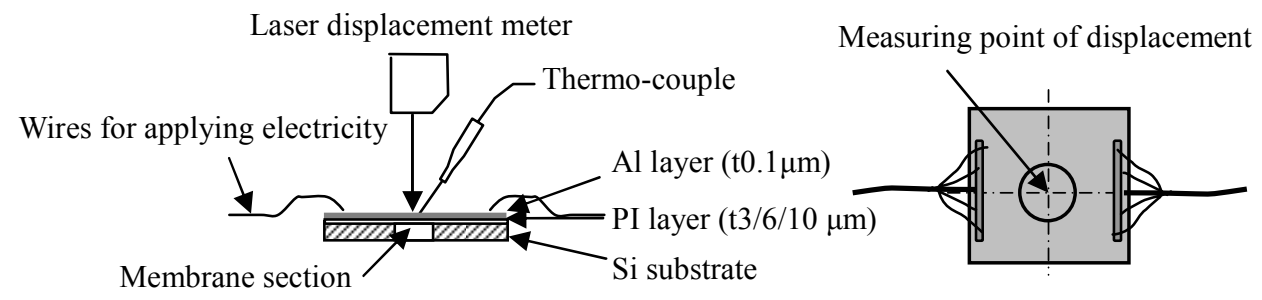

Fig. 9 Setup for measuring membrane deflection. Electricity is applied to aluminum (Al) layer deposited on polyimide (PI) membrane. This causes aluminum layer to thermally expand.

Figure 10 plots the characteristics of the displacement of the membrane center and the temperature of the $\mathrm{Al}$ layer when a continuous voltage of $1 \mathrm{~V}$ was applied. The polyimide membrane was $10-\mu \mathrm{m}$ thick. The membrane produced a maximum displacement of about 11 $\mu \mathrm{m}$ in about $20 \mathrm{~s}$. The displacement sensor indicated that the membrane deflected toward the Al layer side. The displacement slightly decreased over time (-8\% per min). Reproducibility of this tendency was also observed. The reason for this is considered to be the heat transfer from the aluminum layer to the polyimide layer, creep in the polyimide, and so on. Figure 11 plots the relationship between maximum membrane displacement and its thickness when the thickness changes in the experiment of Fig.10. The figure indicates that maximum membrane deflection is approximately inversely proportional to the square root of the membrane thickness.

Next, periodic electricity was applied. Figure 12 plots the membrane deflections when the input voltage was intermittently turned on and off within a period of about $50 \mathrm{~s}$. The figure indicates that the membrane displacement varied corresponding to the temperature of the $\mathrm{Al}$ 
layer and almost constant amplitudes of displacement were reproduced. Thus, this system is able to reproduce displacement on the order of $10 \mu \mathrm{m}$ within a period of several tens of seconds.

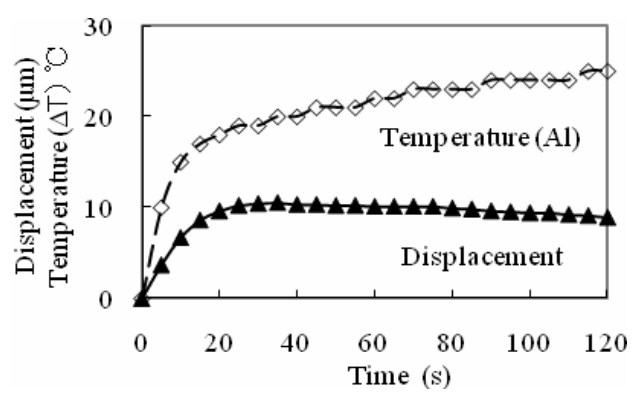

Fig. 10 Relationship between time and measured membrane displacement when continuous voltage of $1 \mathrm{~V}$ is applied.

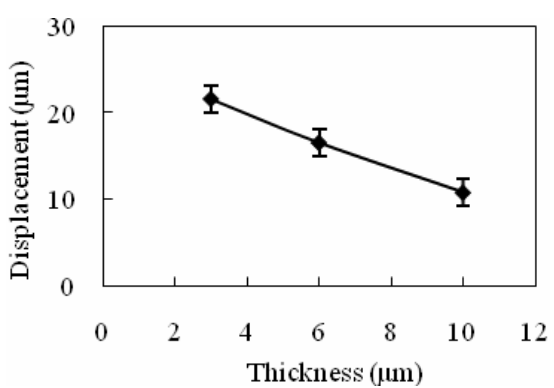

Fig. 11 Relationship between membrane thicknesses and maximum membrane displacement when continuous voltage of $1 \mathrm{~V}$ is applied.

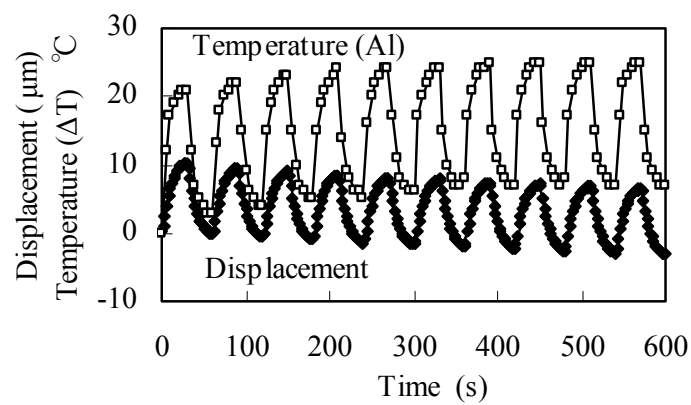

Fig. 12 Displacement of membranes and temperature of aluminum (Al) layer for intermittent electricity input. Applied voltage of $1 \mathrm{~V}$ is turned on and off intermittently within period of about $50 \mathrm{~s}$.

\section{Conclusions}

(1) A fabrication process for thin-film polyimide membranes by DRIE using the Bosch process was proposed. The DRIE was used to etch an Si substrate instead of wet etching. This method has advantage of simple fabricating process and low stiffness of the membrane due to not using an etch-stop layer. The minimum thickness that could be achieved with this method was $2 \mu \mathrm{m}$, which is comparable with that in conventional studies. The experiments applying pressure to the membrane revealed the membranes could produce sufficiently large displacement: the maximum deflections of several hundred micrometers (membrane diameter: $5 \mathrm{~mm}$, thickness: $3-10 \mu \mathrm{m}$ ). Experiments and theoretical analysis suggested that it was necessary to decrease the residual stress in the membrane.

(2) The membranes were actuated by thermal expansion due to electricity being applied. The experiments demonstrated that 3-10 $\mu \mathrm{m}$ thick membranes (diameter: $5 \mathrm{~mm}$ ) could produce deflection in the range from 10-20 $\mu \mathrm{m}$ at the center of the membranes and they could work periodically within a period of several tens of seconds by turning the input on and off. The experiments demonstrated that this system could work as a MEMS actuator. However, it is necessary to improve response performance.

\section{References}

(1) Shearwood, C., Harradine, M., Birch, T., and Stevens, J., Application of polyimide membranes to MEMS technique, Microelectronic Engineering, Vol. 30, Issues 1-4, (1996), pp.547-550.

(2) Nara, K., Nagae, H., Ichiyanagi, M., Jeong, O., and Konishi, S., Investigation of polyimide thin 
film as MEMS material, IEEK, Journal of semiconductor technology and science, Vol. 5, No. 1, (2005), pp.38-44.

(3) Lin, P., Hsieh, H., and Su, G., Design and fabrication of a large-stroke MEMS deformable mirror for wave front control, Journal of Optics, Vol. 13, No.5, (2011), pp.1-8.

(4) Horng, R., Chen, K., Tsai, Y., Suen, C., and Chang, C., Fabrication of a dual-coil dynamic microphone by MEMS techniques, Journal of Micromechanics and Microengineering, Vol. 20, No. 6, (2010), pp.1-7.

(5) Gowrishetty, U., Walsh, K., and Berfield, T., Fabrication of polyimide bi-stable diaphragms using oxide compressive stresses for the field of 'Buckle MEMS', Journal of Micromechanics and Microengineering, Vol. 20, No. 7, (2010), pp.1-6.

(6) Atkinson, G., Pearson, R., Ounaies, Z., Park, C., Harrison, J., et al., Novel piezoelectric polyimide MEMS, Transducers, Solid-State Sensors, Actuators and Microsystems, $12^{\text {th }}$ International Conference 2003 (Transducers'03), Vol. 1, (2003), pp.782-785.

(7) Takeshima, N., and Fujita, H., Polyimide bimorph actuators for a ciliary motion system, 1991 ASME Winter Meeting, DSC-vol.32 (Micromechanical Sensors, actuators, and Systems), (1991), pp.203-209.

(8) Parameswaran, M., Ristic, L., Chau, K., Robinson, A., and Allegretto, W., CMOS electrothermal microactuators, IEEE Micro Electro Mechanical Systems Workshop, (1990), pp.128-131.

(9) Hitachi Chemical DuPont MicroSystems, High heat-resistance fine polyimide for VLSI (in Japanese), Hitachi Chemical DuPont MicroSystems technical report, (2002, 2003).

(10) Kato, W., The basics of applied elasticity (in Japanese), Corona publishing, (1974), pp.127.

(11) Beams, J., Mechanical properties of thin film of gold and silver, The structure and properties and thin film, New York, John Wiley and Sons, (1959), pp.184. 